\title{
Paste structure and rheological properties of lotus seed starch-glycerin monostearate complexes formed by high-pressure homogenization
}

\author{
Bingyan Chen ${ }^{\mathrm{a}}$, Zebin Guo ${ }^{\mathrm{a}}$, Shaoxiao Zeng ${ }^{\mathrm{a}}$, Yuting Tian ${ }^{\mathrm{a}}$, Song Miao ${ }^{\mathrm{a}, \mathrm{b}}$,, Baodong Zheng ${ }^{\mathrm{a}, * *}$ \\ a College of Food Science, Fujian Agriculture and Forestry University, Fuzhou, Fujian 350002, China \\ b Teagasc Food Research Centre, Moorepark, Fermoy, Co. Cork, Ireland
}

\section{A R T I C L E I N F O}

\section{Keywords:}

Lotus seed starch

Glycerin monostearate

High pressure homogenization

Paste structure

Rheological properties

\begin{abstract}
A B S T R A C T
Starch-lipid complexes were prepared using lotus seed starch (LS) and glycerin monostearate (GMS) via a highpressure homogenization (HPH) process, and the effect of HPH on the paste structure and rheological properties of LS-GMS was investigated. Rapid Visco Analyser (RVA) profiles showed that HPH treatment inhibited the formation of the second viscosity peak of the LS-GMS paste, and the extent of this change was dependent on the level of homogenized pressure. Analysis of the size-exclusion chromatography, light microscopy, and low-field ${ }^{1} \mathrm{H}$ nuclear magnetic resonance results revealed that high homogenized pressure (70-100 MPa) decreased molecular weight and size by degrading the branch structure of amylopectin; however, intact LS-GMS granules can optimize the network structure by filler-matrix interaction, which causes free water to transition into immobile water in the starch paste. The steady-shear results showed that the LS-GMS pastes presented non-Newtonian shear-thinning behavior, with higher homogenized pressure producing a smaller hysteresis loop area. During the oscillation process, the LS-GMS pastes prepared at $100 \mathrm{MPa}$ exhibited the lowest loss tangent values in all the complexes, indicating a stronger resistance to vibration.
\end{abstract}

\section{Introduction}

Starch is a commonly consumed carbohydrate, and makes up over $50 \%$ of the daily energy intake in Asian countries (Lesmes, Cohen, Shener, \& Shimoni, 2009). Amylose is one of the main fractions of starch, and is composed of glucose units connected by $\alpha-1,4$ bonds. With heat treatment, amylose can form inclusion complexes with a variety of linear guest molecules, such as fatty acids, emulsifiers, flavor compounds, and phenols (Ali \& Hasnain, 2013; Fanta, Shogren, \& Salch, 1999; Tapanapunnitikul, Chaiseri, Peterson, \& Thompson, 2008; Yue, Chen, Li, \& Mei, 2009). During the formation of these complexes, the hydrophobic part of the guest molecule is incorporated inside the amylose helix because the interior surface of the helix is hydrophobic. The best known inclusion complexes are starch-lipid complexes because of their importance in numerous food applications. Starch-lipid complexes not only contribute significantly toward the functional and nutritional properties of starch, including retarding retrogradation (Tian et al., 2010) and decreasing starch hydrolysis (Kawai, Takato, Sasaki, \& Kajiwara, 2012), but also change the rheological behavior of starch paste (Raphaelides, 2007).

Several studies have found that the addition of lipid can strongly affect the rheology of starch systems. Raphaelides and Georgiadis (2008) showed that the formation of starch-lipid complexes effectively retards the gelation process and reduces the apparent viscosity of starch paste; they also found that the extent of this change was dependent on the chain length of the fatty acids. Fanta, Kenar, Byars, Felker, and Shogren (2010) found that the rheological properties of starch-lipid dispersion can be controlled by adjusting the $\mathrm{pH}$. Meng, Ma, Sun, Wang, and Liu (2014) demonstrated that different types of fatty acid esters exhibited various effects on the rheological properties of starch gel, and also found that hydrophilic fatty acid esters effectively form stable complexes with a high storage modulus and flow behavior index. In recent year, high-pressure homogenization $(\mathrm{HPH})$ appears to be promising for preparing starch-lipid complexes because it is an environment friendly and low-cost method. The high shear employed during $\mathrm{HPH}$ can enhance complex efficiency by improving the dispersion of lipids as well as the level of starch disintegration (Meng et al., 2014; Meng, Ma, Cui, \& Sun, 2015). However, there is insufficient knowledge about how these complexes influence the rheological properties of starch paste.

Lotus (Nelumbo nucifera) is an important aquatic crop that is widely cultivated in China, India, Thailand, Japan, and Australia (Kim \& Shin,

\footnotetext{
* Correspondence to: S. Miao, Teagasc Food Research Centre, Moorepark, Fermoy, Ireland.

** Correspondence to: B. Zheng, College of Food Science, Fujian Agriculture and Forestry University, No.15 Shangxiadian Road, Cangshan District, Fuzhou City, Fujian Province, China.

E-mail addresses: song.miao@teagasc.ie (S. Miao), zbdfst@163.com (B. Zheng).
} 
2012). Besides its popularity as an ornamental flower, lotus seeds are rich in certain types of vitamins and minerals, and lotus seed starch is a popular food ingredient that is processed into various food products in China, such as pastries, fried slices, punching powder, and instant food products. However, native starch has poor solubility, retrogrades easily, and has poor storage stability at low temperatures, and these factors profoundly affect the quality and shelf-life of the end products (Singh, Kaur, \& Mccarthy, 2007). To overcome these drawbacks, various encapsulation techniques have been studied and are currently utilized (Serfert, Drusch, \& Schwarz, 2009; Xu et al., 2013). In our previous study, lotus seed starch-glycerin monostearate complexes were formed at $40 \mathrm{MPa}$ by $\mathrm{HPH}$ treatment and a more stable V-type crystalline complex was formed under 80-100 MPa, which not only changed solubility behaviors but also significantly delayed the short-term retrogradation process of lotus seed starch (Chen et al., 2017). For applying these complexes in the food industry, the paste structure and rheological properties of these complexes are highly important to be analyzed and determined, Rheological properties are closely related to texture attributes, and also contribute to the stability, sensory properties, and consumer acceptability of the end product (Li, Zhu, Guo, Brijs, \& Zhou, 2014). To date, there has been limited research on the paste structure and rheological properties of these complexes.

Therefore, the objective of the present study was to investigate the effect of HPH on the paste structure and rheological properties of a starch-lipid complex prepared using lotus seed starch (LS) and glycerin monostearate (GMS). The paste structure was characterized by the Rapid Visco Analyser (RVA), light microscopy, size exclusion chromatography (SEC), and low-field ${ }^{1} \mathrm{H}$ nuclear magnetic resonance $\left(\mathrm{LF}_{-}{ }^{1} \mathrm{H}\right.$ NMR). The rheological properties, including the steady shear characteristics and the viscoelastic parameters (storage modulus, loss modulus, and loss tangent), were determined. Furthermore, the relationships between the paste structure and the rheological properties of LS-GMS at different pressures were studied and compared.

\section{Materials and methods}

\subsection{Materials}

LS with an amylose/amylopectin ratio of 45/55 was obtained from Green Field Food Co., Ltd. (Fujian, China). The raw starch was defatted by ethanol solution and was dried in an air oven at $40 \pm 1^{\circ} \mathrm{C}$ for $16 \mathrm{~h}$. GMS with a hydrophile-lipophile balance of 3.8 was obtained from TNJ Chemical Industry Co., Ltd. (Hefei, China). All other chemical reagents used in this study were of analytical grade.

\subsection{Formation of the complexes by high-pressure homogenization}

The GMS $(0.5 \mathrm{~g}, 2 \%, w / \mathrm{w}$, dry starch base) was dissolved in $50 \%$ ethanol solution and added to the defatted starch slurry (25 g, 5\%,w/ w) before heating. The mixtures were heated in a water bath at $40{ }^{\circ} \mathrm{C}$ with vigorous stirring for $10 \mathrm{~min}$ to disperse evenly. The mixed slurry was homogenized using a laboratory-scale high-pressure homogenizer (GYB 40-10S; Donghua Technology Co., Ltd., Shanghai, China) at 40, $50,60,70,80,90$, or $100 \mathrm{MPa}$ for five passes. Approximately $500 \mathrm{~mL}$ of the starch-GMS suspension was obtained at each pressure level. After high-pressure treatment, the homogenized sample was incubated at $20{ }^{\circ} \mathrm{C}$ for $12 \mathrm{~h}$. Samples were collected by centrifugation $(4500 \times \mathrm{g}$, $10 \mathrm{~min}$ ) and washed twice with a 50\% water/ethanol mixture to remove any uncomplexed GMS. The final precipitates (LS-GMS) were freeze-dried and ground with a laboratory-scale grinder until able to pass through a 100-mesh sieve. As a control, a sample without GMS was also prepared.

\subsection{Rapid Visco Analyser}

The pasting properties of the LS and LS-GMS were determined using an RVA (Perten Scientific Instruments Ltd., Stockholm, Sweden). LS-GMS (2.5 g, dry basis) and distilled water were mixed and stirred in an aluminum RVA sample container to produce an $8.0 \mathrm{wt} \%$ slurry. The slurry was first kept at $50{ }^{\circ} \mathrm{C}$ for $1 \mathrm{~min}$, heated to $95^{\circ} \mathrm{C}$ within $5 \mathrm{~min}$, kept at $95{ }^{\circ} \mathrm{C}$ for $10 \mathrm{~min}$, cooled $50{ }^{\circ} \mathrm{C}$ within $5 \mathrm{~min}$, and subsequently kept at $50{ }^{\circ} \mathrm{C}$ for $2 \mathrm{~min}$. The paddle speed was $960 \mathrm{rpm}$ in the beginning $10 \mathrm{~s}$ to disperse the sample, and then the paddle speed was set at $160 \mathrm{rpm}$ during the measurement. This test was performed in triplicate, and all measurements were analyzed by TCW software (Perten Scientific Instruments Ltd., Stockholm, Sweden).

\subsection{Size-exclusion chromatography}

The molecular weight of LS and LS-GMS pastes were measured using the method reported by Zeng et al. (2015). An aliquot of the filtered sample $(300 \mu \mathrm{L})$ was subjected to HPSEC-MALLS-RI (Wyatt Technology, Santa Barbara, CA, USA) after passing through a $0.22-\mu \mathrm{m}$ nylon filter. The HPSEC system consisted of P8514-806 columns (Showa Denko, Tokyo, Japan), MALLS (632.8 nm; DAWN DSP; Wyatt Technology), and an RI detector (Optilab DSP; Wyatt Technology). The mobile phase consisted of dimethyl sulfoxide with $50 \mathrm{mmol} / \mathrm{L} \mathrm{LiBr}$; the flow velocity was $0.35 \mathrm{~mL} / \mathrm{min}$, and the $\mathrm{dn} / \mathrm{dc}$ value was set at 0.074 . Further, the molecular weight $\left(M_{w}\right)$, mean square radius $\left(R_{z}\right)$, and polydispersity $(\mathrm{Mw} / \mathrm{Mn})$ of the starch pastes were determined by the designated software.

\subsection{Amylose leaching}

The LS and LS-GMS pastes were prepared by RVA. $2 \mathrm{~g}$ of samples was accurately weighed and suspended in $20 \mathrm{~mL}$ of distilled water, and the sample was then centrifuged $(3000 \times g, 10 \mathrm{~min})$. The leaching of amylose from the suspension was determined according to the iodine color value method (Juliano et al., 2010). Amylose (E. Merck, Darmstadt, Germany) from corn starch (E. Merck) was used to generate the standard curve.

\subsection{Light microscopy}

The LS and LS-GMS pastes were prepared by RVA and were viewed under $400 \times$ magnification using a multi-functional optical microscope (BX-43FS1; Olympus, Tokyo, Japan) after staining with $0.2 \%$ iodine solution.

\section{7. $L F-{ }^{1} H N M R$}

The proton transversal relaxation time was measured used a 23MHz NMR analyzer (NMI20-015 V-I; Niumag, Co., Ltd., Shanghai, China). Approximately $1 \mathrm{~g}$ of the LS-GMS complex paste prepared by RVA was transferred to a $15-\mathrm{mm}$ diameter NMR glass tube. All measurements were performed at $25 \pm 0.1^{\circ} \mathrm{C} . \mathrm{T}_{2}$ measurements were performed using a sequence based on the Carr-Purcell-Meiboom-Gill (CPMG) sequence. Typical pulse parameters were as follows: the echo time was $400 \mu \mathrm{s}$; the echo number was set to 8000 , the waiting time was $8 \mathrm{~s}$, and eight scans were acquired for each measurement. Relaxation curves obtained from the CPMG sequence were analyzed using the exponential model [Eq. (1)].

$\mathrm{S}(\mathrm{t})=\sum \mathrm{A}_{\mathrm{i}} \exp \left(-\mathrm{t} / \mathrm{T}_{2 \mathrm{i}}\right)$

\subsection{Rheological measurements}

The rheological properties of the LS and LS-GMS pastes were assessed using a rotational rheometer (Physical MCR 301; Anton Paar, Co., Ltd., Stuttgart, Germany) with a parallel plate sensor $(60 \mathrm{~mm}$ diameter, $1 \mathrm{~mm}$ gap). The temperature was maintained at $25{ }^{\circ} \mathrm{C}$ during 
these rheological measurements.

\subsubsection{Steady flow measurements}

The steady flow tests were programmed to linearly increase from 1.0 to $300 \mathrm{~s}^{-1}$ over $3 \mathrm{~min}$, followed immediately by a linear decrease from 300 to $1.0 \mathrm{~s}^{-1}$. The experimental data of the flow curve were obtained and fitted using the Herschel-Bulkley model (Guo et al., 2015).

$\tau=\tau_{0}+K \gamma^{\mathrm{n}}$

\subsubsection{Frequency sweep measurements}

Frequency sweep tests were performed over the frequency range of $0.1-10 \mathrm{~Hz}$, with constant deformation $(0.5 \%$ strain) within the linear viscoelastic range. The $0.5 \%$ strain was within the linear viscoelastic region according to the strain sweep results (data not shown). The mechanical spectra were obtained, which recorded the storage modulus $\left(\mathrm{G}^{\prime}\right)$, loss modulus $\left(\mathrm{G}^{\prime \prime}\right)$, and loss tangent $\left(\tan \delta=\mathrm{G}^{\prime \prime} / \mathrm{G}^{\prime}\right)$ as functions of the frequency $(\mathrm{Hz})$.

\subsection{Statistical analysis}

All measurements were performed in triplicate, and graphs were constructed using Origin Pro 8.5. The data were analyzed, and significant differences were determined by DPS 9.05 (SciencePress, Beijing, China). $P \leq 0.05$ was considered to be statistically significant.

\section{Results and discussion}

\subsection{Pasting properties}

The pasting properties of LS and LS-GMS at different homogenized pressures were shown in Fig. 1A and B. LS showed the highest peak

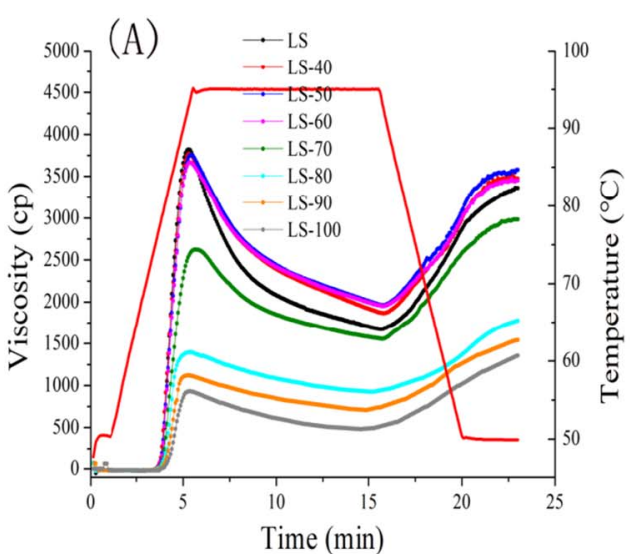

(C)

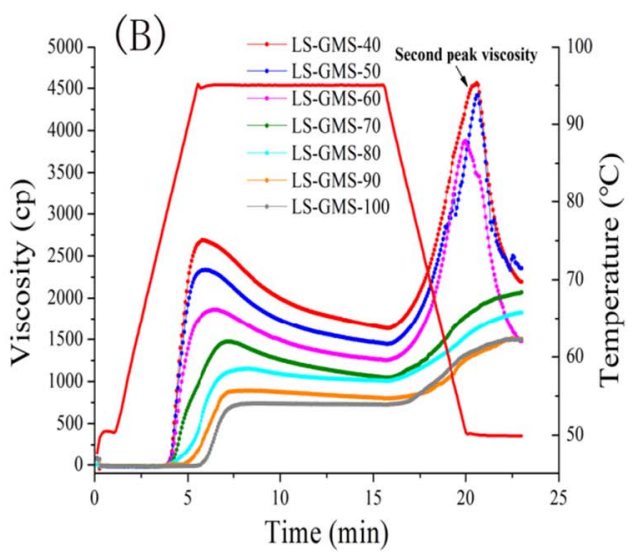

(D)
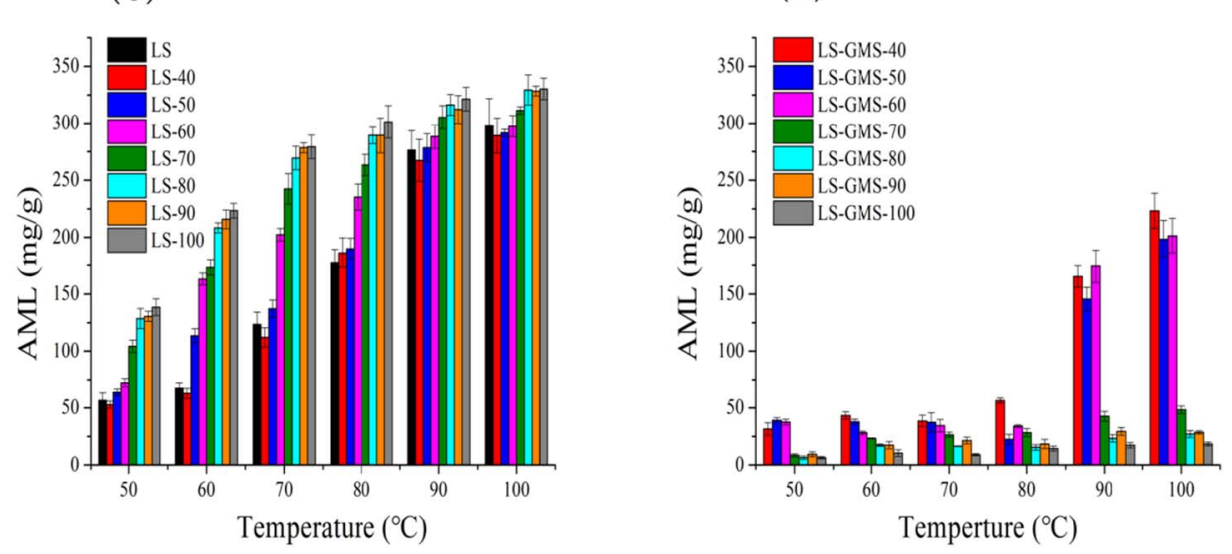

viscosity and breakdown viscosity in all samples, which was result from the swelling and disruption of starch granules in gelatinization (Fig. 1A). Compared with LS, no obvious changes in pasting profile occurred at 40-60 MPa, however, the peak viscosity of LS decreased significantly when the pressure beyond $60 \mathrm{MPa}$. This result could be attributed to the restructuring that occurs during HPH treatment. Upon high pressure homogenization, highly branched amylopectin can produce partially degraded dextrin with a high proportion of short chains, which is not ideal for the swelling and further decreases the peak viscosity of starch paste (Yang, Chaib, Gu, \& Hemar, 2016). When GMS was added to starch system in HPH process, LS-GMS samples delayed pasting and decreased the peak viscosity (Fig. 1B). The peak viscosity of the LS-GMS pastes decreased with increasing homogenized pressure, reaching the minimum value ( $849 \mathrm{cp}$ ) at $100 \mathrm{MPa}$, which was $15.34 \%$ lower compared to control lacking GMS. This result is in line with the findings from a study by Gerits, Pareyt, and Delcour (2015), who reported that amylose-lipid complexes result in an insoluble film on the surface of the starch granules that impedes their swelling, thus weak gelatinization process. Additionally, the second viscosity peak was observed in the LS-GMS pastes at 40-60 MPa during the cooling phase. When the homogenized pressure beyond $60 \mathrm{MPa}$, the second viscosity peak of the LS-GMS paste disappeared. The LS-GMS pastes at 70-100 MPa exhibited a decrease in the breakdown viscosity and setback viscosity as the homogenized pressure increased. According to the previous studies (Chang, He, Fu, Huang, \& Jane, 2014; Karkalas, Ma, Morrison, \& Pethrick, 1995), amylose complexes can be divided into two separable state: less ordered type-I complexes and semi-crystalline type-II complexes due to their thermodynamic characteristics. Type-II complexes have a more crystalline structure and dissociates at a temperature $>105^{\circ} \mathrm{C}$. The formation of the second viscosity peak can mainly be attributed to the dissociation of the unstable type-I complex during gelatinizaton. Sufficient levels of amylose, lipid, and amylopectin resulted in the formation of a new gel network during the cooling

Fig. 1. The RVA profiles (A and B) and AML (C and D) of LS and LS-GMS prepared at different homogenized pressures during gelatinization. 
Table 1

The molecular structure (A) and water distribution (B) of gelatinized LS and LS-GMS prepared by HPH treatment.

(A) The molecule structure of gelatinized LS and LS-GMS prepared by HPH treatment.

\begin{tabular}{|c|c|c|c|c|}
\hline & $M_{w}\left(10^{6} \mathrm{~g} / \mathrm{mol}\right)$ & $M_{n}\left(10^{6} \mathrm{~g} / \mathrm{mol}\right)$ & $R_{\mathrm{z}}(\mathrm{nm})$ & $M_{w} / M_{n}$ \\
\hline LS & $8.17 \pm 0.22^{\mathrm{a}}$ & $2.53 \pm 0.13^{\mathrm{ab}}$ & $168.4 \pm 2.3^{\mathrm{a}}$ & $3.23 \pm 0.11^{\mathrm{ab}}$ \\
\hline LS-40 & $8.10 \pm 0.20^{\mathrm{ab}}$ & $2.43 \pm 0.23^{\mathrm{b}}$ & $163.2 \pm 0.8^{\mathrm{b}}$ & $3.33 \pm 0.03^{\mathrm{a}}$ \\
\hline LS-50 & $7.92 \pm 0.04^{\mathrm{b}}$ & $2.67 \pm 0.06^{\mathrm{a}}$ & $160.1 \pm 1.3^{\mathrm{c}}$ & $2.97 \pm 0.23^{b c}$ \\
\hline LS-60 & $7.63 \pm 0.16^{c}$ & $2.58 \pm 0.08^{\mathrm{ab}}$ & $151.2 \pm 1.6^{\mathrm{d}}$ & $2.95 \pm 0.23^{b c}$ \\
\hline LS-70 & $4.53 \pm 0.12^{\mathrm{d}}$ & $1.64 \pm 0.02^{c}$ & $120.6 \pm 1.3^{\mathrm{e}}$ & $2.76 \pm 0.23^{\mathrm{c}}$ \\
\hline LS-80 & $2.54 \pm 0.05^{\mathrm{e}}$ & $1.12 \pm 0.02^{\mathrm{d}}$ & $91.3 \pm 1.3^{\mathrm{f}}$ & $2.63 \pm 0.23^{\mathrm{cd}}$ \\
\hline LS-90 & $2.25 \pm 0.07^{\mathrm{f}}$ & $0.85 \pm 0.05^{\mathrm{e}}$ & $86.4 \pm 1.2^{g}$ & $2.64 \pm 0.23^{c}$ \\
\hline LS-100 & $2.17 \pm 0.07^{g}$ & $0.95 \pm 0.07^{\mathrm{de}}$ & $85.1 \pm 1.2^{\mathrm{g}}$ & $2.28 \pm 0.23^{\mathrm{d}}$ \\
\hline LS-GMS-40 & $8.06 \pm 0.12^{\mathrm{a}}$ & $2.66 \pm 0.21^{\mathrm{a}}$ & $162.1 \pm 1.3^{\mathrm{a}}$ & $3.03 \pm 0.11^{\mathrm{a}}$ \\
\hline LS-GMS-50 & $7.84 \pm 0.11^{\mathrm{ab}}$ & $2.51 \pm 0.15^{\mathrm{ab}}$ & $161.3 \pm 1.3^{\mathrm{a}}$ & $3.12 \pm 0.06^{\mathrm{a}}$ \\
\hline LS-GMS-60 & $7.74 \pm 0.19^{\mathrm{b}}$ & $2.58 \pm 0.11^{\mathrm{a}}$ & $158.7 \pm 1.4^{\mathrm{b}}$ & $3.08 \pm 0.03^{\mathrm{a}}$ \\
\hline LS-GMS-70 & $4.76 \pm 0.08^{c}$ & $2.34 \pm 0.10^{b}$ & $135.8 \pm 1.4^{c}$ & $2.02 \pm 0.06^{\mathrm{b}}$ \\
\hline LS-GMS-80 & $2.36 \pm 0.15^{\mathrm{d}}$ & $1.16 \pm 0.11^{\mathrm{d}}$ & $92.3 \pm 1.3^{\mathrm{d}}$ & $2.04 \pm 0.12^{\mathrm{b}}$ \\
\hline LS-GMS-90 & $2.33 \pm 0.11^{\mathrm{d}}$ & $1.34 \pm 0.10^{\mathrm{cd}}$ & $91.6 \pm 1.1^{\mathrm{d}}$ & $1.74 \pm 0.03^{\mathrm{c}}$ \\
\hline LS-GMS-100 & $2.28 \pm 0.09^{d}$ & $1.48 \pm 0.12^{\mathrm{c}}$ & $91.5 \pm 1.1^{\mathrm{d}}$ & $1.47 \pm 0.07^{\mathrm{d}}$ \\
\hline
\end{tabular}

(B) Internal water distribution of LS and LS-GMS treated with gelatinization.

\begin{tabular}{|c|c|c|c|c|c|c|}
\hline & \multicolumn{3}{|c|}{ Time of relaxation(ms) } & \multicolumn{3}{|l|}{ W (\%) } \\
\hline & $\mathrm{T}_{21}$ & $\mathrm{~T}_{22}$ & $\mathrm{~T}_{23}$ & $\mathrm{~A}_{21}$ & $\mathrm{~A}_{22}$ & $\mathrm{~A}_{23}$ \\
\hline LS & $1.14-1.74$ & $21.54-71.64$ & ND. & $2.36 \pm 0.34^{\mathrm{f}}$ & $97.64 \pm 1.18^{\mathrm{a}}$ & ND. \\
\hline LS-40 & $0.49-0.86$ & $14.17-98.34$ & ND & $1.57 \pm 0.17^{\mathrm{g}}$ & $98.43 \pm 1.34^{\mathrm{a}}$ & ND \\
\hline LS-50 & $1.74-3.51$ & $37.64-100.00$ & $100.00-132.22$ & $4.82 \pm 0.15^{\mathrm{d}}$ & $91.97 \pm 1.32^{\mathrm{b}}$ & $3.21 \pm 0.08^{\mathrm{e}}$ \\
\hline LS-60 & $1.74-6.13$ & $32.75-100.00$ & $100.00-200.92$ & $7.54 \pm 0.12^{\mathrm{a}}$ & $80.59 \pm 1.12^{\mathrm{c}}$ & $11.87 \pm 0.03^{\mathrm{d}}$ \\
\hline LS-70 & $2.01-4.64$ & $24.77-100.00$ & $100.00-705.31$ & $5.87 \pm 0.06^{\mathrm{bc}}$ & $27.37 \pm 1.64^{\mathrm{d}}$ & $66.76 \pm 1.54^{\mathrm{c}}$ \\
\hline LS-80 & $0.49-5.33$ & $49.72-100.00$ & $100.00-1232.65$ & $6.21 \pm 0.26^{\mathrm{b}}$ & $22.38 \pm 1.73^{\mathrm{e}}$ & $71.41 \pm 1.98^{\mathrm{b}}$ \\
\hline LS-90 & $1.32-2.01$ & $49.77-100.00$ & $100.00-1873.82$ & $3.26 \pm 0.27^{\mathrm{e}}$ & $16.43 \pm 1.40^{\mathrm{f}}$ & $80.31 \pm 0.87^{\mathrm{a}}$ \\
\hline LS-100 & $0.57-1.51$ & $21.54-100.00$ & $100.00-3274.55$ & $5.43 \pm 0.70^{c}$ & $14.43 \pm 0.13^{5}$ & $80.14 \pm 0.79^{\mathrm{a}}$ \\
\hline LS-GMS-40 & $1.14-2.65$ & $57.22-100.00$ & $100.00-2154.37$ & $2.89 \pm 0.17^{\mathrm{b}}$ & $20.28 \pm 1.25^{\mathrm{e}}$ & $76.83 \pm 1.54^{\mathrm{a}}$ \\
\hline LS-GMS-50 & $1.32-2.65$ & $57.22-100.00$ & $100.00-932.19$ & $4.37 \pm 0.21^{\mathrm{a}}$ & $20.67 \pm 2.06^{\mathrm{e}}$ & $74.96 \pm 1.88^{\mathrm{b}}$ \\
\hline LS-GMS-60 & $0.75-1.32$ & $43.28-100.00$ & $100.00-613.28$ & $1.78 \pm 0.18^{\mathrm{d}}$ & $30.27 \pm 1.67^{\mathrm{d}}$ & $67.95 \pm 1.23^{\mathrm{c}}$ \\
\hline LS-GMS-70 & $0.57-1.00$ & $37.64-100.00$ & $100.00-305.21$ & $2.33 \pm 0.09^{c}$ & $43.48 \pm 2.01^{\mathrm{c}}$ & $54.19 \pm 1.40^{\mathrm{d}}$ \\
\hline LS-GMS-80 & $0.49-0.86$ & $43.28-100.00$ & $100.00-280.53$ & $3.06 \pm 0.11^{\mathrm{b}}$ & $53.27 \pm 1.36^{\mathrm{b}}$ & $43.67 \pm 2.32^{\mathrm{e}}$ \\
\hline LS-GMS-90 & $0.49-0.75$ & $57.77-100.00$ & $100.00-185.31$ & $4.67 \pm 0.05^{\mathrm{a}}$ & $55.62 \pm 1.52^{\mathrm{ab}}$ & $39.71 \pm 1.27^{\mathrm{f}}$ \\
\hline LS-GMS-100 & $0.43-0.75$ & $57.12-100.00$ & $100.00-152.32$ & $3.13 \pm 0.05^{\mathrm{b}}$ & $57.36 \pm 1.34^{\mathrm{a}}$ & $39.51 \pm 0.89^{f}$ \\
\hline
\end{tabular}

$M w$ : weight-average molecular mass; $M n$ : number-average molecular mass; $R z$ : radius of gyration; $M w / M n$ : polydispersity.

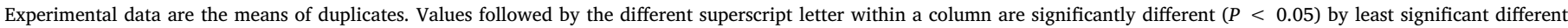
(LSD) test. ND means no detected.

phase, which increased the setback viscosity of the starch paste. However, the gel network may be imperfect and easily damaged during the stirring by the RVA, thus reducing the final viscosity. The RVA profiles of LS-GMS suggest that high homogenized pressure can increase thermal stability of complexes and inhibit the formation of the second viscosity peak, thereby further changing the pasting structure of LS-GMS.

\subsection{Molecular weight distribution}

In order to provide useful information on the characteristics of the polymeric chains in the starch paste, the molecular weight of the LS and LS-GMS pastes at different homogenized pressure were determined by HPSEC-MALLS-RI; the results are summarized in Table 1A. The $M_{w}$ and $R_{z}$ of the LS paste were $8.17 \times 10^{6} \mathrm{~g} / \mathrm{mol}$ and $168.4 \mathrm{~nm}$, respectively, which are both several times more than those of other samples because of the presence of amylopectin, which is highly branched (Zhang, Zeng, Wang, Zeng, \& Zheng, 2014). Following HPH treatment, LS and LS-GMS displayed similar change trend in molecular weight distribution, and no obvious difference was found between molecular weight of LS and LSGMS at same homogenized pressure. Low homogenized pressure (40-60 MPa) had no obvious impact on the $M_{w}$ and $R_{z}$ of starch samples. Conversely, after HPH treatment at $80 \mathrm{MPa}$, the $M_{w}$ and $R_{z}$ of LS sharply decreased to $2.54 \times 10^{6} \mathrm{~g} / \mathrm{mol}$ and $91.3 \mathrm{~nm}$, respectively; the
$M_{w}$ and $R_{z}$ of LS-GMS sharply decreased to $2.36 \times 10^{6} \mathrm{~g} / \mathrm{mol}$ and $92.3 \mathrm{~nm}$, respectively. Furthermore, less dramatic changes were observed after further increasing the pressure to $100 \mathrm{MPa}$. These results indicated that the molecular weight of LS-GMS pastes were mainly dependent on homogenized pressure rather than complex reaction. High shear forces can break polymer chains by disrupting covalent bonds and that the rate of degradation is dependent on the amount of mechanical energy exerted. This result was also consistence with the previous study (Putseys, Lamberts, \& Delcour, 2010), who reported that starch complex reaction is a process from amylose inclusion helix to supramolecular sarch assemblies, and has little impacted on molecule structure. The polydispersity $\left(M_{w} / M_{n}\right)$, which is an indication of the narrowness of the distribution, of amylopectin in the LS-GMS pastes ranged from 3.03 to 1.47 across the entire homogenized pressures. When the homogenized pressure reached $100 \mathrm{MPa}$, the $M_{w} / M_{n}$ ratio of the LS-GMS paste was close to 1, which was lower than that of LS-100, suggested that the molecule weight distribution of LS-GMS-100 paste trend toward homogeneity. According to a previous study (Meng et al., 2014), starch-palmitic complexes with $4-5 \mu \mathrm{m}$ of particle diameter were formed in $100 \mathrm{MPa}$ of $\mathrm{HPH}$ treatment. The narrow molecule weight distribution of LS-GMS-100 may be attributed to the formation of short-chain starch complexes, that contribute more orderly semicrystalline structure. 


\subsection{Amylose leaching and optical microscopy}

Amylose leaching (AML) is an important feature of paste structure. When starch granules are gradually heated in excessive water, the granules swell, and the amylose molecules leach into the supernatant, resulting in the formation of a gel network. Studies on AML have shown the extent of the interaction among the starch chains in amorphous starch paste (Zeng et al., 2016). The extent of AML in the LS and LS-GMS in gelatinization was shown in Fig. 1C and D. The extent of AML in LS increased with increasing temperature due to the severe destruction of granule integrity. Following HPH treatment, the AML extent increased with the increase of homogenized pressure in starch granule. It is in accordance with the report by Che et al. (2007), the shear stress could enhance swelling and therefore promote the gelatinization of starch granules. On the other hand, the extent of AML in the LS-GMS samples was much lower than that in LS. This result could be attributed to the formation of the complexes in starch granules, which inhibits AML in the supernatant. Moreover, when the temperature was over $90^{\circ} \mathrm{C}$, LS-GMS at 40-60 MPa displayed a sharp rise in the extent of AML, presumably due to the complex dissociated and released free amylose at high temperatures. The extent of AML in LS-GMS showed a decreasing trend with increasing homogenized pressure. At the end of the gelatinization process, the amount of AML in LS-GMS at $100 \mathrm{MPa}$ was $23.47 \mathrm{mg} / \mathrm{mL}$, which was $89.80 \%$ lower than that of LS-GMS at $40 \mathrm{MPa}$. These results are in agreement with those of our previous study (Chen et al., 2017), which indicated that homogenized pressure within the range of $40-60 \mathrm{MPa}$ is insufficient for inducing the formation of stable type-II complexes with long-chain fatty acids, and that high homogenized pressure can improve the thermal stability of these complexes, resulting in less AML. The extent of AML in the LS-GMS pastes was also assessed by microscopy with diluted iodine, as shown in Fig. 2. For controls lacking GMS, all the starch granules swelled dramatically, and extensive AML was observed in the homogeneous mixture, which stained blue in the image. However, when GMS was added into starch system in HPH process, LS-GMS adopted different images in gelatinization. With an increase in the homogenized pressure, LS-GMS displayed more irregular transparent crystals that were stained pale red, and only a few instances of AML were observed in the image. This result further confirmed that high homogenized pressure is favorable for the formation of stable complexes, which restrain AML during gelatinization.

\subsection{Water mobility in the gelatinized starch complex}

The spin-spin relaxation time $\left(\mathrm{T}_{2}\right)$ is particularly sensitive to changes in molecular mobility, and reflects the difference in the degrees of freedom of water (Lu et al., 2011). The water in food matrices can be classified into three fractions according to the water mobility: bound water $\left(\mathrm{T}_{21}: 1-10 \mathrm{~ms}\right)$, immobile water $\left(\mathrm{T}_{22}: 10-100 \mathrm{~ms}\right)$, and free water $\left(\mathrm{T}_{23}: 100-1000 \mathrm{~ms}\right)$. A higher degree of freedom is associated with a longer transverse relaxation time (Rnm \& Gamr, 2003). The proton transverse relaxation times $\left(\mathrm{T}_{2}\right)$ for the LS and LS-GMS pastes at different homogenized pressure were determined by LF- ${ }^{1} \mathrm{H}$ NMR, and their $\mathrm{T}_{2}$ distributions are shown in Fig. 3. LS displayed two separate water fractions that could be assigned to bound water $\left(\mathrm{T}_{21}=1.14-1.74 \mathrm{~ms}\right)$ and immobile water $\left(\mathrm{T}_{22}=21.54-75.64 \mathrm{~ms}\right)$ in the starch gel, respectively. Little free water was observed in the LS paste because of the high levels of AML in gelatinization, which strengthens the interaction
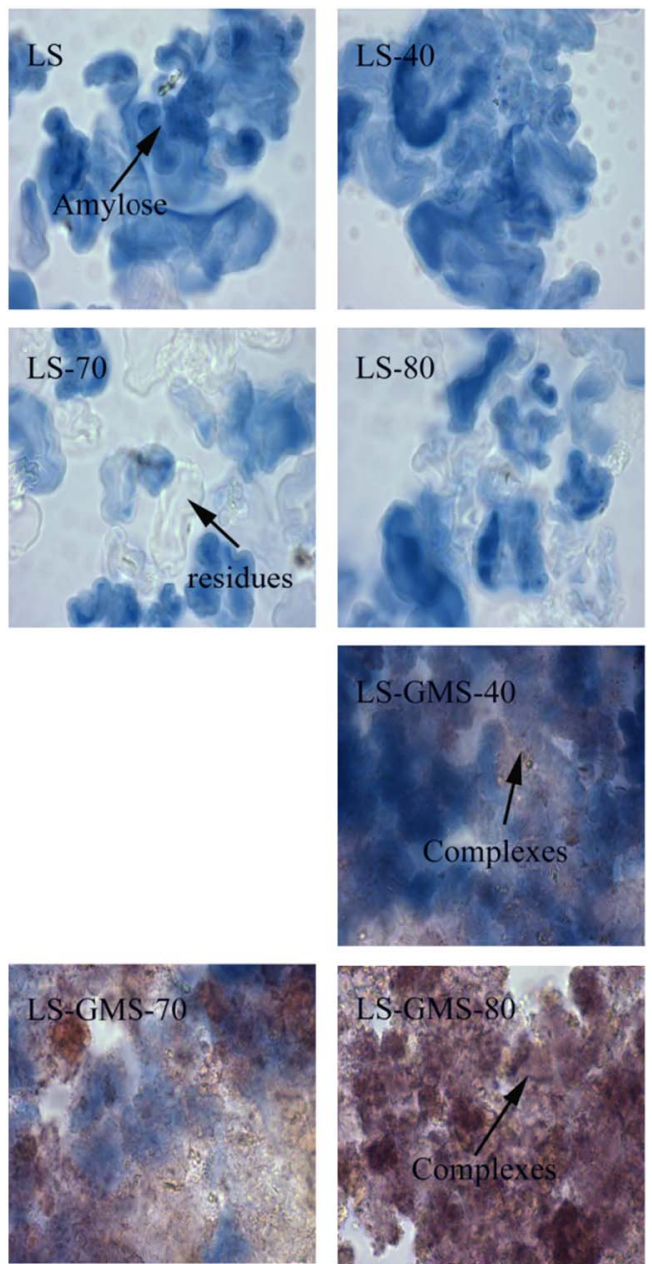
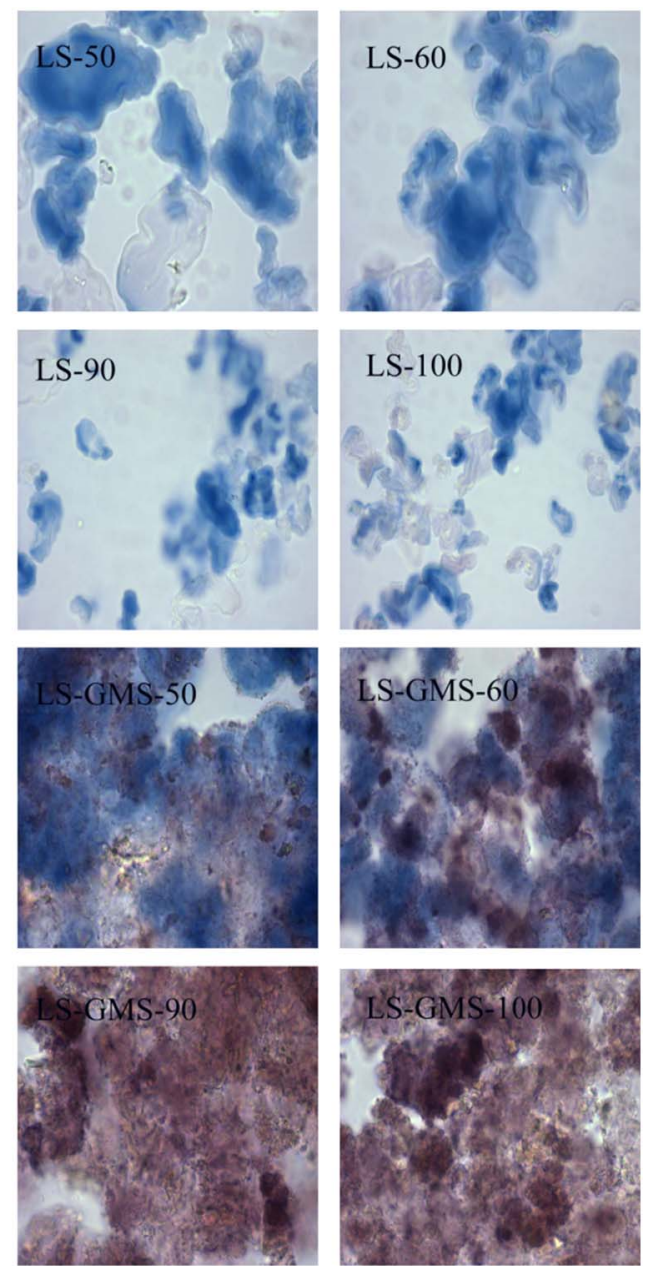

Fig. 2. Light micrographs $(400 \times)$ of gelatinized LS and LS-GMS prepared at different homogenized pressures. (For interpretation of the references to color in this figure, the reader is referred to the web version of this article.) 

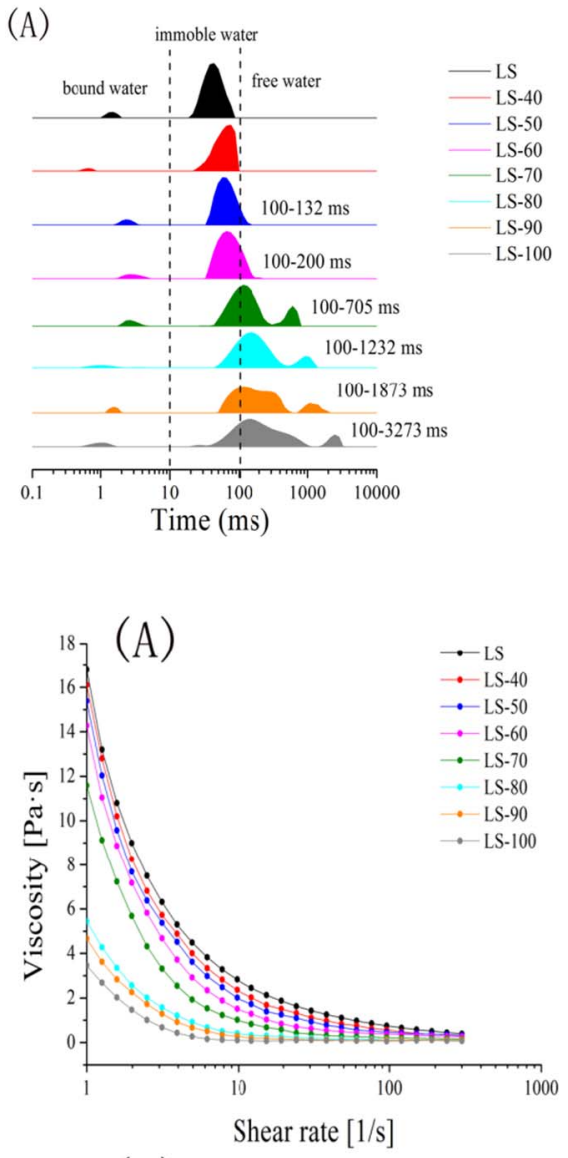

(C)

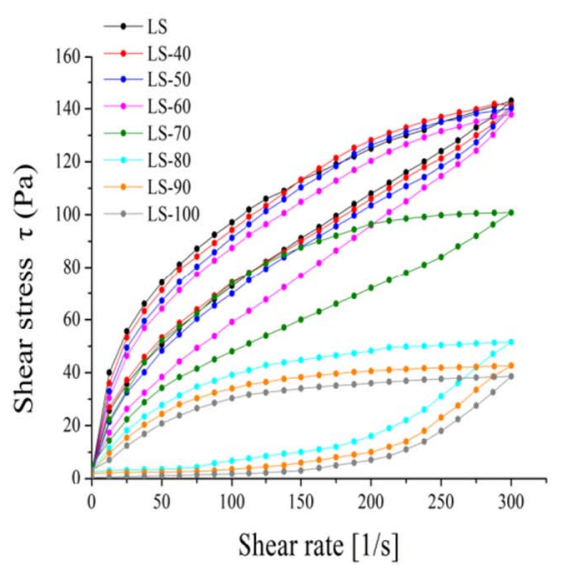

(B)
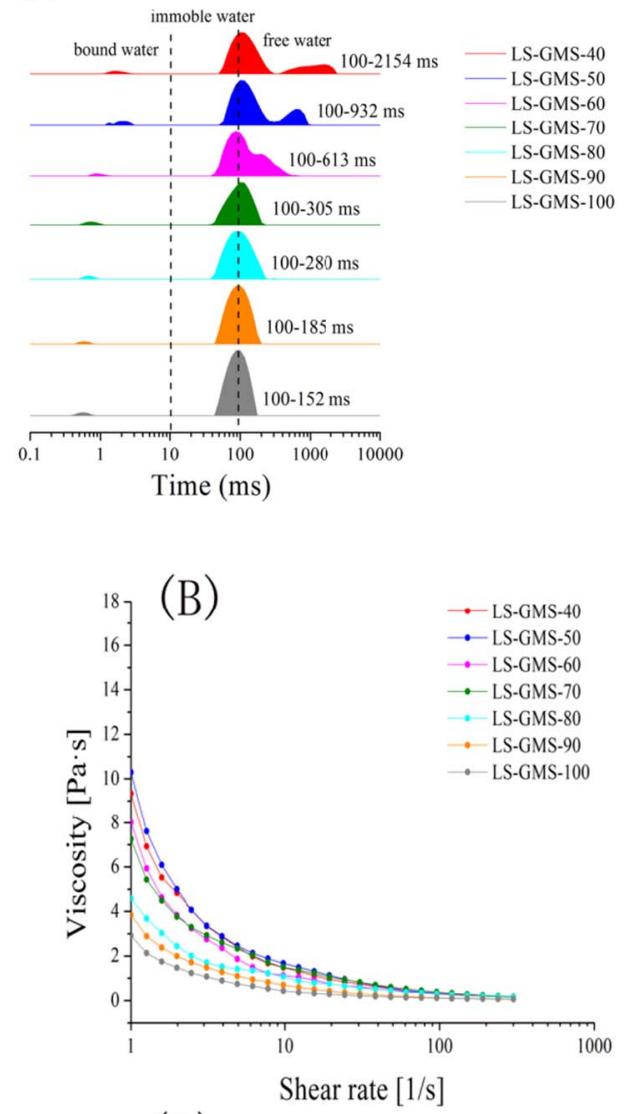

(D)

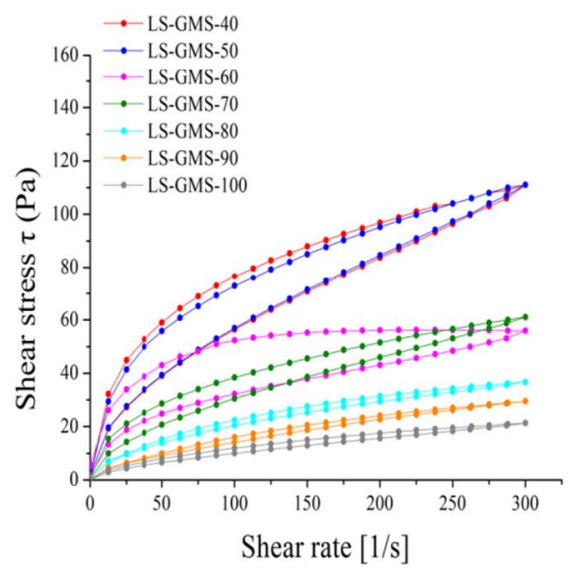

Fig. 3. Low-field ${ }^{1} \mathrm{H}$ NMR of gelatinized LS and LS-GMS prepared at different homogenized pressures.
Fig. 4. Flows behavior of gelatinized LS and LS-GMS prepared at different homogenized pressures. between amylopectin and water molecule, thus further enhancing the water-holding capacity of the starch gel. Compared to the water distribution of LS paste, an additional water fraction $\left(\mathrm{T}_{23}>100 \mathrm{~ms}\right)$ with higher mobility was found in LS pastes at 70-100 MPa, which indicates the interactions was weakened and the presence of free water in the homogenized-starch paste system. When GMS was added into the starch system in HPH process, LS-GMS showed a wide free water distribution range from $100 \mathrm{~ms}$ to $2100 \mathrm{~ms}$, the proton transverse relaxation time of free water fraction $\left(\mathrm{T}_{23}\right)$ declined with increasing homogenized pressure. The results showed a tendency for these two water fractions (free water and immobile water) to merge together with increasing homogenized pressure in the LS-GMS samples. At $100 \mathrm{MPa}$, the LS-GMS pastes showed a narrow $\mathrm{T}_{2}$ distribution range (57.12-152.32 ms), indicating the freedom of water molecule decreased and more homogeneous water distribution in the LS-GMS system.
According to the results reported by Tang, Godward, and Hills (2001), the relative peak areas $\left(A_{2}\right)$ of the relaxation spectra were proportional to the relative water content. The different water fractions in the LS-GMS paste system are quantitatively summarized in Table 1B. At $40-60 \mathrm{MPa}$, LS-GMS showed a high relative peak area in $\mathrm{A}_{23}$, revealing a higher content of free water in the LS-GMS paste. This result can be attributed to the fact that free lipid derived from the dissociation of LS-GMS can weaken the plastic effect of water linked to starch polymer chains through hydrogen bonds, further leading to the formation of an imperfect gel network during the cooling phase and thereby reducing the water-holding capacity of starch gel. Furthermore, $\mathrm{A}_{23}$ decreased and $\mathrm{A}_{22}$ increased with increasing homogenized pressure, which suggests that high homogenized pressure can change water distribution of LS-GMS paste, resulting in a certain amount of free water shifting to immobile water. 


\subsection{Rheological properties}

\subsubsection{Flow behavior}

The flow curves of the LS and LS-GMS pastes at different homogenized pressure were shown in Fig. 4. All the starch samples exhibited non-Newtonian shear-thinning flow behavior (Fig. 4A, B) because the apparent viscosity of the samples decreased within the shear rate range of $0-300 \mathrm{~s}^{-1}$. Similar results for starch-polysaccharide systems have been previously reported (Chen, Tong, Ren, \& Zhu, 2014). Additionally, the apparent viscosity of LS and LS-GMS decreased with increasing homogenized pressure. At $80 \mathrm{MPa}$, LS and LS-GMS paste showed much lower apparent viscosity as a function of the shear rate. However, when the homogenized pressure was further increased, the continuous decrease in the apparent viscosity was smaller. The apparent viscosity of starch pastes is a flow behavior caused by molecular chain entanglement that restricts molecular motion (Mohd, Azemi, \& Manan, 1999). Nayouf, Loisel, and Doublier (2003) reported that highly branched amylopectin and long chains of amylose are fractured during HPH treatment, resulting in increased flexibility in the movement of the starch molecules in the solution. Thus, it is reasonable to assume that the sharp decline in the apparent viscosity of the starch paste at $80 \mathrm{MPa}$ can be ascribed to the degradation of the starch molecules caused by various mechanical forces during HPH treatment, such as shearing, cavitation, and collision. While there is a definite minimum to the chain length during the HPH process (Wang, Li, Wang, Liu, \& Adhikari, 2012), the homogenized energy was insufficient to further disrupt the chain structure with increase of the pressure to $100 \mathrm{MPa}$. This could be why the decrease in the apparent viscosity was not proportional to the homogenized pressure. This result is consistent with the results of the change in molecular weight (Table 1A), which showed lower molecular weight and smaller molecular size of the starch fraction formed after $\mathrm{HPH}$ treatment at 70-100 MPa.

The Herschel-Bulkley model [Eq. (1)] was applied to describe the variation in the flow behavior of the starch paste system. The data from the steady flow test for the starch samples fitted well with the Herschel-Bulkley equation $\left(R^{2}>0.99\right.$; Table 2). As shown in Table 2, all the starch samples showed pseudoplastic flow behavior when $n$ was $<1$. The value of $n$ increased with increasing homogenized pressure for both the up and down curves, whereas the $K$ value generally showed a decreasing trend. This result indicates that the flow behavior of all starch paste has a tendency to move toward that of a Newtonian fluid during shear process. Additionally, all the flow curves displayed a distinct hysteresis loop, as shown in Fig. 4C and D. The thixotropic property of the starch pastes could be ascribed to the damage of the gel network structure caused by continuous shearing (Richardson, Kidman, Langton, \& Hermansson, 2004). The hysteresis loop area of LS increased with the increase of homogenized pressure, indicating the easy damage of starch gel network caused by shear after HPH treatment. Moreover, when GMS was added into starch system in HPH process, the hysteresis loop area of LS-GMS decreased with the increase of homogenized pressure. At 70-100 MPa, the LS-GMS paste had a smaller hysteresis loop area, indicating that the time-dependent thinning flow behavior of starch paste was weaken due to the formation of amylose - GMS at high homogenized pressure.

\subsubsection{Dynamic rheological properties}

Dynamic modulus is known to be a good characterization tool in studying the interaction between dispersed phase and continuous phase in some polymeric solutions (Pongsawatmanit, Temsiripong, Ikeda, \& Nishinari, 2006). Changes in the storage modulus ( $\left.G^{\prime}\right)$, loss modulus $\left(\mathrm{G}^{\prime \prime}\right)$ and loss tangent $(\tan \delta)$ as functions of frequency are shown in Fig. 5. For the LS paste, the storage modulus, G', was significantly greater than the loss modulus, $G^{\prime \prime}$, which indicates a strong gel structure. The $G^{\prime}$ and $G^{\prime \prime}$ showed a significant decrease for LS paste when subjected to HPH treatment at $70 \mathrm{MPa}$, which could be attributed to the disruption of gel network in high pressure. Moreover, addition of 

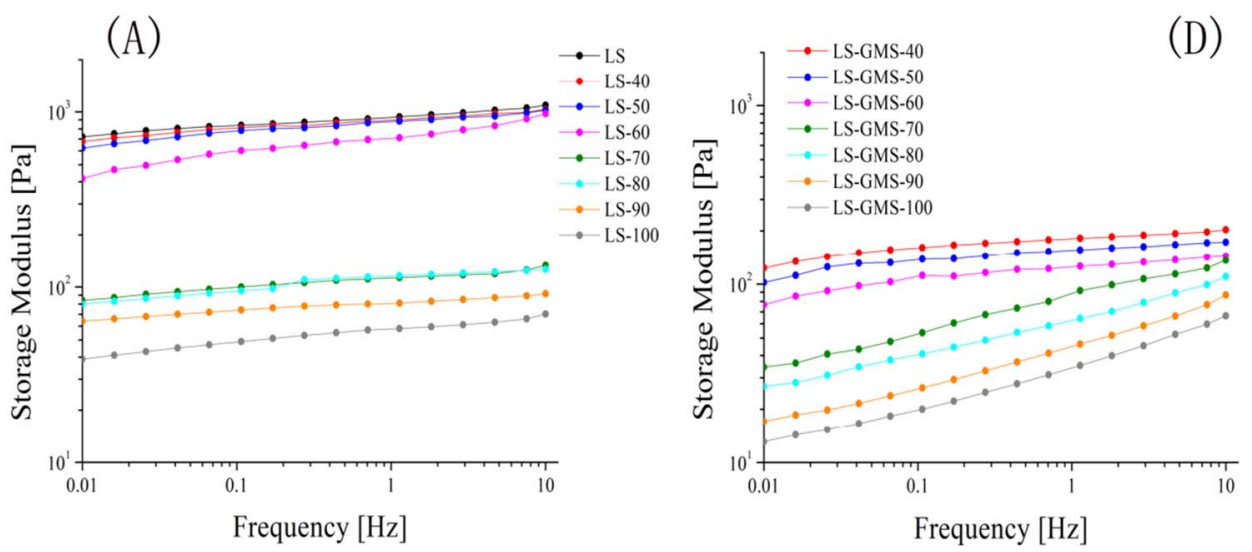

Fig. 5. The viscoelastic properties of gelatinized LS and LS-GMS prepared at different homogenized pressures.

(B)

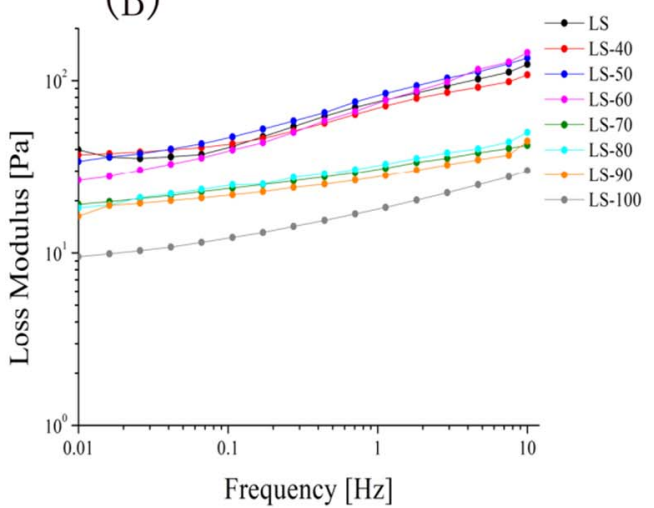

(C)

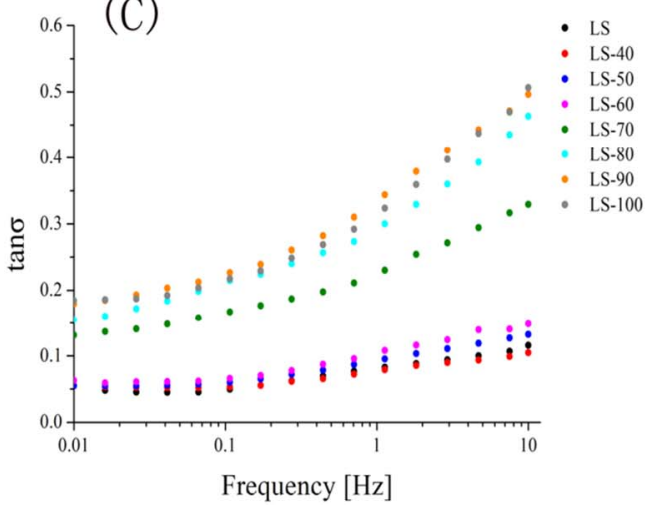

(E)

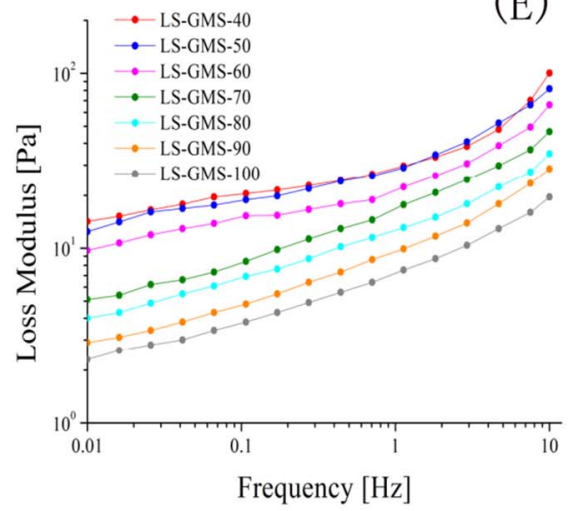

$(\mathrm{F})$

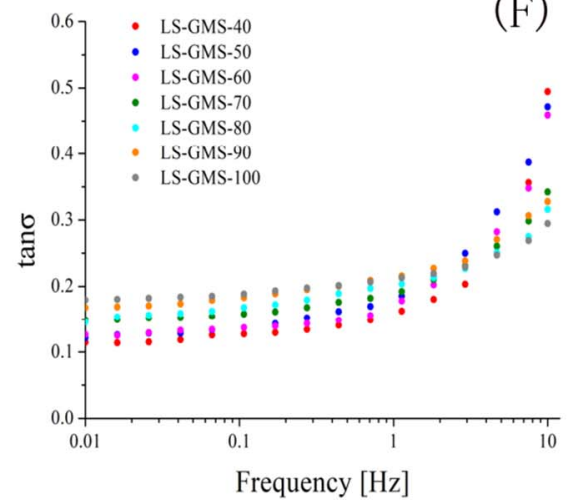

GMS further decreased $\mathrm{G}^{\prime}$ and G" of starch paste during HPH treatment. Similarly, gelling behavior was also observed in a previous study on bambara starch-lipid complexes (Oyeyinka, Singh, Ma, \& Amonsou, 2016), which indicated that the presence of complexes that hampered the swelling of starch granules, thus further slowing the formation of the gel junction zone between the starch molecules.

The loss tangent $(\tan \delta)$ is an indicator of viscoelastic properties, and is calculated as the ratio of $\mathrm{G}^{\prime \prime}$ to $\mathrm{G}^{\prime}$. Tan $\delta$ can be used to study the change in viscoelastic properties caused by ingredient interaction such as junction zone disruption, which results in a sudden rise in $\tan \delta$ (Chakrabarti, Gaonkar, \& Mcpherson, 2006). As shown in Fig. 5C and F, all the starch samples displayed predominantly solid-like behaviors (tan $\delta<1$ ) over the frequency range from $0.1-10 \mathrm{~Hz}$; The LS paste showed the lowest initial $\tan \delta$ value in all the starch samples, indicating the formation of a strong gel network in the amorphous region. The $\tan \delta$ value of LS paste was remarkably increased at the high pressure range (70-100 MPa), indicating that high pressurization weakens the junction zones of starch gel of LS paste. It can be also found that the initial $\tan \delta$ value for LS-GMS paste increased with the increase of homogezation pressure, which is in agreement with the modulus results $\left(G^{\prime}, G^{\prime \prime}\right)$, further demonstrated that the rigid structure of starch paste can be weaken after the formation of complexes However, it should be noted that LS-GMS paste displayed a reverse trend in the final $\tan \delta$ values compared with LS, and lowest final $\tan \delta$ value was observed in LS-GMS paste at $100 \mathrm{MPa}$. These results suggested that high homogenized pressure could improve the vibration resistance of the LS-GMS pastes, thus retarding the change in the starch paste network form being more solid-like to a more viscous gel during oscillation process.

\subsection{Relationships among the paste structure and rheological properties}

Starch gelatinization is defined by the swelling and melting of the crystalline structure of starch granules. When starch granules are heated in excess water, the starch that leaches out, which is mainly amylose, creates a fairly regular gel network by forming a number of cross-links in the continuous phase (RindlavWestling, Mats Stading, \& Gatenholm, 2002), as shown in Fig. 6A. Many studies have reported on the influence of complexation on starch pasting and 

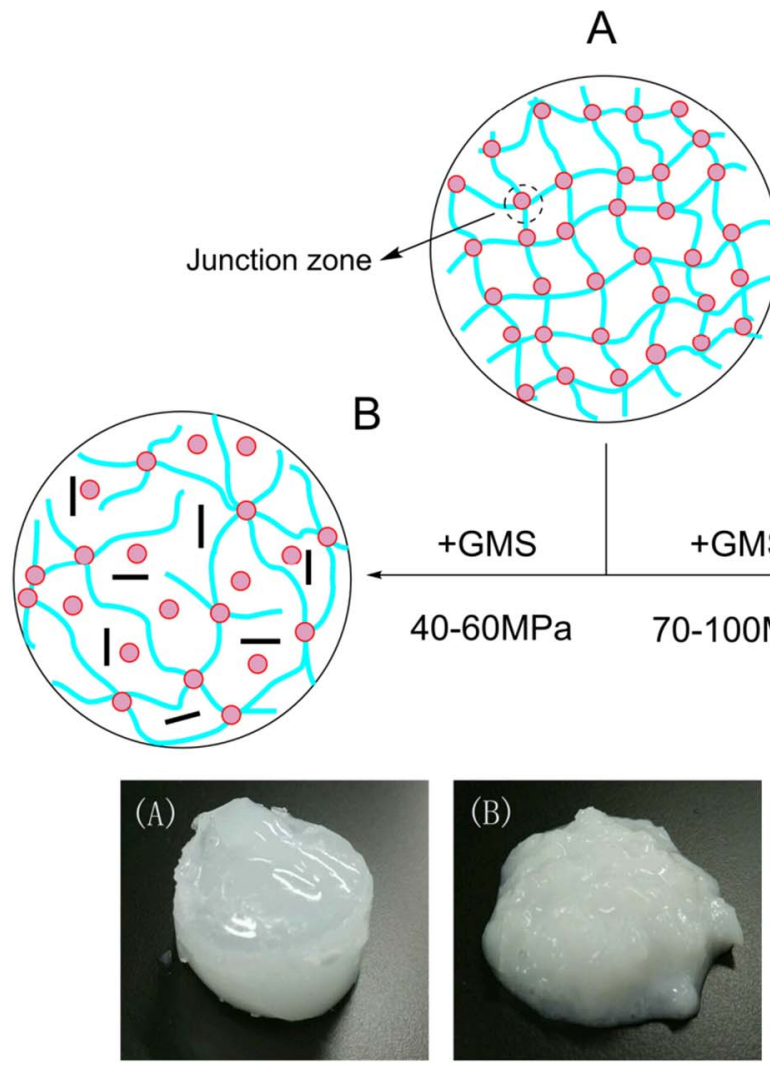

Amylopectin

Amylose

\section{GMS}

LS-GMS

\section{Filler-matrix interaction}

Fig. 6. Schematic illustration of the network structure in gelatinized LS-GMS prepared at different homogenized pressures: (A) LS; (B) 40-60 MPa: (C) 70-100 MPa. rheological properties. In our study, when GMS added into the starch system in the homogenization process, the paste structure and rheological properties of LS-GMS were clearly changed by HPH treatment. Molecular structure and molecular behavior changes were shown to be responsible for the changes in the rheological properties. At 40-60 MPa, there were no obvious changes in the molecular structure of LS-GMS. However, amylose-GMS complexes can significantly degrade and release more free GMS at high temperatures, which is not ideal for junction zone formation during the cooling process; this process was confirmed by the RVA profiles and AML results (Fig. 1). Weaker junction zones can decrease water-holding capacity and result in the formation of a fragile network, as shown in Fig. 6B, which showed high loss tangent during oscillation process.

High homogenized pressure (70-100 MPa) can decrease the degree of amylopectin branching and increase the amount of short linear molecular chains, which can increase amylose-amylose interaction. Consequently, intermolecular entanglement was reduced, which further resulted in significantly lower apparent viscosity and viscoelasticity $\left(\mathrm{G}^{\prime}\right.$, $\left.\mathrm{G}^{\prime \prime}\right)$ of the pastes. These results are in agreement with those of previous studies, which reported that molecular chain structure has a critical relationship with rheological properties (Lewandowska, 2015; Zhong et al., 2015). On the other hand, molecular behavior plays an important role in modifying rheological properties. The rheological properties of starch gel would be determined not only by the density of spatial entanglements between amylopectin in the continuous phase but also by the effective contact of ghosts, which are the elastic structures heterogeneously distributed within the continuous viscous matrix (Ratnayake \& Jackson, 2007). High homogenized pressure reduces AML, which is the origin of the dissociation of inclusion complex in gelatinization. More intact inclusion complexes, which were observed under microscopy, could act as deformable fillers, and were embedded in the junction zone of the continuous matrix. This further optimized the starch-water network structure and improved the vibration resistance of the paste, leading to decreases in the hysteresis loop area and loss tangent. This result has also been confirmed by the LF- ${ }^{1} \mathrm{H}$ NMR studies; with an increase in the homogenized pressure, the amount of immobile water increases and the water distribution in the starch paste becomes more uniform, thus promoting the formation of a homogeneous paste (Fig. 6C).

\section{Conclusion}

In this study, we investigated the influence of HPH treatment on the paste structure and rheological properties of LS-GMS. The results indicated that the RVA pasting profiles of LS-GMS are evidently affected by HPH treatment, and the extent of this change was dependent on the level of homogenized pressure applied. HPH treatment at 70-100 MPa reduced the amount of AML in gelatinization, and inhibited the formation of the second viscosity peak. Homogenized pressure was the key factor determining the molecular structure of the LS-GMS paste. High homogenized pressure has the ability to decrease intermolecular entanglement by degrading the amylopectin, leading to a decline in the apparent viscosity and viscoelasticity. Additionally, we found that LS-GMS plays an important role in the filler-matrix interaction. Intact LS-GMS granules can act as deformable fillers, and were embedded in the junction zone of the continuous matrix, which not only changed the starch gel network, but also optimized water distribution, thus further delaying the oscillatory response. This study provides important insight into the flow behavior of the lotus seed product incorporating GMS under gelatinization.

\section{Acknowledgments}

This study was financially supported by the National Natural Science Foundation of China (No. 31501485) and the Scientific and Technological Innovation Team Support Plan of Institution of Higher Learning in Fujian Province (Grant No. [2012]03). The study was also supported by the Construction Projects of Top University at Fujian Agriculture and Forestry University of China (Grant No. 612014042). 


\section{References}

Ali, T. M., \& Hasnain, A. (2013). Effect of emulsifiers on complexation and retrogradation characteristics of native and chemically modified White sorghum (Sorghum bicolor) starch. Thermochimica Acta, 552, 46-53.

Chakrabarti, S., Gaonkar, A. G., \& Mcpherson, A. (2006). Probing ingredient functionalities in food systems using rheological methods. Ingredient interactions effects on food quality. 65. Ingredient interactions effects on food quality (pp. 119-125).

Chang, F., He, X., Fu, X., Huang, Q., \& Jane, J. (2014). Effects of heat treatment and moisture contents on interactions between lauric acid and starch granules. Journal of Agricultural and Food Chemistry, 62, 7862.

Che, L., Li, D., Wang, L., Özkan, N., Chen, X. D., \& Mao, Z. (2007). Effect of high-pressure homogenization on the structure of cassava starch. International Journal of Food Properties, 10, 911-922.

Chen, B., Zeng, S., Zeng, H., Guo, Z., Zhang, Y., \& Zheng, B. (2017). Properties of lotus seed starch-glycerin monostearin complexes formed by high pressure homogenization. Food Chemistry, 226, 119-127.

Chen, L., Tong, Q., Ren, F., \& Zhu, G. (2014). Pasting and rheological properties of rice starch as affected by pullulan. International Journal of Biological Macromolecules, 66, 325-331.

Fanta, G. F., Kenar, J. A., Byars, J. A., Felker, F. C., \& Shogren, R. L. (2010). Properties of aqueous dispersions of amylose-sodium palmitate complexes prepared by steam jet cooking. Carbohydrate Polymers, 81, 645-651.

Fanta, G. F., Shogren, R. L., \& Salch, J. H. (1999). Steam jet cooking of high-amylose starch-fatty acid mixtures. An investigation of complex formation. Carbohydrate Polymers, 38, 1-6.

Gerits, L. R., Pareyt, B., \& Delcour, J. A. (2015). Wheat starch swelling, gelatinization and pasting: Effects of enzymatic modification of wheat endogenous lipids. LWT - Food Science and Technology, 63, 361-366.

Guo, Z., Zeng, S., Zhang, Y., Lu, X., Tian, Y., \& Zheng, B. (2015). The effects of ultra-high pressure on the structural, rheological and retrogradation properties of lotus seed starch. Food Hydrocolloids, 44, 285-291.

Juliano, B. O., Perez, C. M., Blakeney, A. B., Castillo, T., Kongseree, N., Laignelet, B., ... Webb, B. D. (2010). International cooperative testing on the amylose content of milled rice. Starch - Stärke, 33, 157-162.

Karkalas, J., Ma, S., Morrison, W. R., \& Pethrick, R. A. (1995). Some factors determining the thermal properties of amylose inclusion complexes with fatty acids. Carbohydrate Research, 268, 233-247.

Kawai, K., Takato, S., Sasaki, T., \& Kajiwara, K. (2012). Complex formation, thermal properties, and in-vitro digestibility of gelatinized potato starch-fatty acid mixtures. Food Hydrocolloids, 27, 228-234.

Kim, M. J., \& Shin, H. S. (2012). Antioxidative effect of lotus seed and seedpod extracts. Food Science and Biotechnology, 21, 1761-1766.

Lesmes, U., Cohen, S. H., Shener, Y., \& Shimoni, E. (2009). Effects of long chain fatty acid unsaturation on the structure and controlled release properties of amylose complexes. Food Hydrocolloids, 23, 667-675.

Lewandowska, K. (2015). Influence of molecular weight on structure and rheological properties of microcrystalline chitosan mixtures. International Journal of Biological Macromolecules, 79, 583-586.

Li, M., Zhu, K. X., Guo, X. N., Brijs, K., \& Zhou, H. M. (2014). Natural additives in wheatbased pasta and noodle products: Opportunities for enhanced nutritional and functional properties. Comprehensive Reviews in Food Science and Food Safety, 13, 347-357.

Lu, S., Chen, J. J., Chen, Y. K., Lii, C. Y., Lai, P., \& Chen, H. H. (2011). Water mobility, rheological and textural properties of rice starch gel. Journal of Cereal Science, 53, $31-36$.

Meng, S., Ma, Y., Cui, J., \& Sun, D. W. (2015). Preparation of corn starch-fatty acid complexes by high-pressure homogenization. Starch - Stärke, 66, 809-817.

Meng, S., Ma, Y., Sun, D. W., Wang, L., \& Liu, T. (2014). Properties of starch-palmitic acid complexes prepared by high pressure homogenization. Journal of Cereal Science, 59, 25-32.

Mohd, N. I., Azemi, B. M. N. M., \& Manan, D. M. A. (1999). Rheological behaviour of sago (Metroxylon sagu) starch paste. Food Chemistry, 64, 501-505.

Nayouf, M., Loisel, C., \& Doublier, J. L. (2003). Effect of thermomechanical treatment on the rheological properties of crosslinked waxy corn starch. Journal of Food Engineering, 59, 209-219.

Oyeyinka, S. A., Singh, S., Ma, Y., \& Amonsou, E. O. (2016). Influence of high-pressure homogenization on the physicochemical properties of bambara starch complexed with lysophosphatidylcholine. LWT - Food Science and Technology, 74, 120-127.

Pongsawatmanit, R., Temsiripong, T., Ikeda, S., \& Nishinari, K. (2006). Influence of tamarind seed xyloglucan on rheological properties and thermal stability of tapioca starch. Journal of Food Engineering, 77, 41-50.

Putseys, J. A., Lamberts, L., \& Delcour, J. A. (2010). Amylose-inclusion complexes: Formation, identity and physico-chemical properties. Journal of Cereal Science, 51, 238-247.

Raphaelides, S. N. (2007). Viscoelastic behavior of amylose-fatty acid gels. Journal of Texture Studies, 23, 297-313.

Raphaelides, S. N., \& Georgiadis, N. (2008). Effect of fatty acids on the rheological behaviour of pea starch dispersions during heating. Food Hydrocolloids, 21, 1188-1200.

Ratnayake, W. S., \& Jackson, D. S. (2007). A new insight into the gelatinization process of native starches. Carbohydrate Polymers, 67, 511-529.

Richardson, G., Kidman, S., Langton, M., \& Hermansson, A. M. (2004). Differences in amylose aggregation and starch gel formation with emulsifiers. Carbohydrate Polymers, 58, 7-13.

RindlavWestling, A., Mats Stading, A., \& Gatenholm, P. (2002). Crystallinity and morphology in films of starch, amylose and amylopectin blends. Biomacromolecules, 3, 84-91.

Rnm, P., \& Gamr, L. (2003). Nuclear magnetic resonance and water activity in measuring the water mobility in Pintado (Pseudoplatystoma corruscans) fish. Journal of Food Engineering, 58, 59-66.

Serfert, Y., Drusch, S., \& Schwarz, K. (2009). Chemical stabilisation of oils rich in longchain polyunsaturated fatty acids during homogenisation, microencapsulation and storage. Food Chemistry, 113, 1106-1112.

Singh, J., Kaur, L., \& Mccarthy, O. J. (2007). Factors influencing the physico-chemical, morphological, thermal and rheological properties of some chemically modified starches for food applications-A review. Food Hydrocolloids, 21, 1-22.

Tang, H. R., Godward, J., \& Hills, B. (2001). The distribution of water in native starch granules-A multinuclear NMR study. Carbohydrate Polymers, 43, 375-387.

Tapanapunnitikul, O., Chaiseri, S., Peterson, D. G., \& Thompson, D. B. (2008). Water solubility of flavor compounds influences formation of flavor inclusion complexes from dispersed high-amylose maize starch. Journal of Agricultural and Food Chemistry, $56,220-226$.

Tian, Y., Yang, N., Li, Y., Xu, X., Zhan, J., \& Jin, Z. (2010). Potential interaction between $\beta$-cyclodextrin and amylose-lipid complex in retrograded rice starch. Carbohydrate Polymers, 80, 581-584.

Wang, B., Li, D., Wang, L. J., Liu, Y. H., \& Adhikari, B. (2012). Effect of high-pressure homogenization on microstructure and rheological properties of alkali-treated highamylose maize starch. Journal of Food Engineering, 113, 61-68.

Xu, J., Zhao, W., Ning, Y., Bashari, M., Wu, F., Chen, H., ... Zhang, L. (2013). Improved stability and controlled release of $\omega 3 / \omega 6$ polyunsaturated fatty acids by spring dextrin encapsulation. Carbohydrate Polymers, 92, 1633-1640.

Yang, Z., Chaib, S., Gu, Q., \& Hemar, Y. (2016). Impact of pressure on physicochemical properties of starch dispersions. Food Hydrocolloids, 68, 164-177.

Yue, W., Chen, Z., Li, X., \& Mei, L. (2009). Effect of tea polyphenols on the retrogradation of rice starch. Food Research International, 42, 221-225.

Zeng, S., Chen, B., Zeng, H., Guo, Z., Lu, X., Zhang, Y., \& Zheng, B. (2016). Effect of microwave irradiation on the physicochemical and digestive properties of lotus seed starch. Journal of Agricultural and Food Chemistry, 64, 95-110.

Zeng, S., Wu, X., Lin, S., Zeng, H., Lu, X., Zhang, Y., \& Zheng, B. (2015). Structural characteristics and physicochemical properties of lotus seed resistant starch prepared by different methods. Food Chemistry, 186, 213-222.

Zhang, Y., Zeng, H., Wang, Y., Zeng, S., \& Zheng, B. (2014). Structural characteristics and crystalline properties of lotus seed resistant starch and its prebiotic effects. Chemical Reviews, 155, 311-318.

Zhong, K., Zhang, Q., Tong, L., Liu, L., Zhou, X., \& Zhou, S. (2015). Molecular weight degradation and rheological properties of schizophyllan under ultrasonic treatment. Ultrasonics Sonochemistry, 23, 75-80. 